\title{
Morphological diagram of diffusion driven aggregate growth in plane: competition of anisotropy and adhesion
}

\author{
A.Yu. Menshutin ${ }^{\mathrm{a}, \mathrm{b}}$, L.N. Shchur ${ }^{\mathrm{a}, \mathrm{b}}$ \\ ${ }^{a}$ Landau Institute for Theoretical Physics, Akademika Semenova av 1-A, Chernogolovka, Moscow Region, 142432, Russia \\ ${ }^{b}$ Scientific Center in Chernogolovka, Institutsky 8, Chernogolovka, Moscow Region, 142432, Russia
}

\section{Abstract}

Two-dimensional structures grown with Witten and Sander algorithm are investigated. We analyze clusters grown offlattice and clusters grown with antenna method with $N_{f p}=3,4,5,6,7$ and 8 allowed growth directions. With the help $\underset{D}{D}$ of variable probe particles technique we measure fractal dimension of such clusters $D(N)$ as a function of their size $N$. We propose that in the thermodynamic limit of infinite cluster size the aggregates grown with high degree of anisotropy $\left(N_{f p}=3,4,5\right)$ tend to have fractal dimension $D$ equal to $3 / 2$, while off-lattice aggregates and aggregates with lower anisotropy $\left(N_{f p}>6\right)$ have $D \approx 1.710$. Noise-reduction procedure results in the change of universality class for DLA. For high enough noise-reduction value clusters with $N_{f p} \geq 6$ have fractal dimension going to $3 / 2$ when $N \rightarrow \infty$.

\section{Introduction}

Understanding critical properties of non-equilibrium processes [1] is still a challenging problem of contemporary statistical physics. Dynamical growth phenomena is of particular interest, both theoretical and practical. The model for the two-dimensional growth (Diffusion Limited Aggregation) was introduced about thirty years ago by Witten and Sander 2] and analytical solution of the model is still a challenging problem. Structures generated by DLA algorithm look very similar to those found in nature and society. Among examples of such structures are [3]: ice crystals on window, mineral dendrites on the surfaces of limestone, colony of bacteria, nano-size crystals grown on the crystal surface, monolayer polymer films, interfaces in Hele-Shaw cell, urban growth, etc. Similarity of such objects leads to the natural questions: are the properties of all above mentioned various structures the same? Are the clusters generated by different models identical or not?

Growth structures are characterized by fractal dimension [4. According to the universality concept of critical phenomena the value of fractal dimension is one of the basic characteristics of universality class (see, f.e., ref. [5]). The technical difficulties in estimation of fractal dimension in DLA model are the high level of fluctuations 6 and strong finite-size effects. To overcome these difficulties we use previously developed approach of fractal dimension measurement 7 based on varying the size of probe particles for calculating the harmonic measure averages and then taking the limit of probe particle size going to zero. This procedure smooths out oscillating dependence of effective fractal dimension on cluster size and allows us to estimate its asymptotic behavior with good accuracy.

Email address: may@itp.ac.ru (A.Yu. Menshutin)
It will be shown in section 4 that such procedure in some sense increases effective cluster size. Therefore, the resulting values of DLA properties calculated as averages over harmonic measure are closer to the thermodynamic limit.

In this paper we present our study of the off-lattice DLA model with two additional parameters, the local field which brakes spatial isotropy and the adhesion coefficient. In Section 2 we present details of algorithm used to grow off-lattice aggregates, it is followed in Section 3 by description of modifications of the algorithm that allow us to build lattice clusters with off-lattice algorithm. In Section 4 we provide details on numerical procedure of our variable probe particles method. In Section 5 we present results of analysis. In Section 6 we propose morphological diagram. We conclude paper with discussion in Section 7.

\section{Off-lattice killing-free algorithm}

We use off-lattice killing free algorithm for cluster generation, developed in [6, 7]. In this algorithm, particles are hard balls of unit size which move in 2D in random direction with fixed step length. Since motion is actually off-lattice one can use some tricks to speed up the algorithm, e.g. particles could jump with large step length if they are far away from the cluster. Another trick that helps us to speed up the simulation is the so-called killingfree technique. When it happens that particle goes far enough from the cluster, i.e. it is outside of circle of radius $R_{r}$ (the return radius, which is larger than radius of the cluster), we return this particle back to the birth circle $R_{b}, R_{b}<R_{r}$. Since in $2 \mathrm{D}$ random motion there is zero probability to escape to infinity, particle will always return to the origin, i.e. any particle will eventually collide with 
the cluster. Therefore, it is physically not correct to kill particles that have come out of some circle $R_{d}$ (the death radius, which is used in traditional DLA algorithm), even if $R_{d} \gg R_{b}$ since it will introduce distortion in the harmonic measure. One can analytically solve Laplace equation and find angle-dependent probability for such particles to intersect $R_{b}$ for the first time at any point. Probability for a particle starting its walk outside circle of radius $R_{b}$ from position $\left(0, R_{0}\right)$ to intersect $R_{b}$ at angle $\phi$ for a first time is given by formula

$$
P(\phi)=\frac{x^{2}-1}{2 \pi\left(x^{2}-2 x \cos \phi+1\right)}, x=R_{0} / R_{b}
$$

In contrast to $2 \mathrm{D}$, in higher dimensions there exists the non zero non-return probability for the random walker. This means that computational model of DLA or any similar process should take this fact into account (see paper [8] with discussion of this subject in 3D).

The algorithm we use is as follows 1

1. Set seed particle at the origin at position $(0,0)$.

2. Calculate size of the cluster $R_{\max }$. Set birth radius value to $R_{b}=R_{\max }+5 R$ and set return radius value to $R_{r}=\left(R_{b}+200 R\right) * 1.2$.

3. Create new particle at random position on the birth radius $R_{b}$

4. Calculate distance $d$ from current particle position to the cluster. If $d<5 R$ than move the particle in random direction with step length equal to $R$ and check for collisions with the cluster, otherwise $(d>$ $5 R$ ) move the particle in random direction with step length equal to $d-5 R$.

5 . If the particle is out of $R_{r}$ than return it back to $R_{b}$ with angle-dependent probability $P(\phi)$ given by formula (1).

6. Repeat steps (4)-(5) until the particle has been attached to the cluster, then start with step (2).

Effective realization of step (4) of the algorithm requires a lot of effort. First, to find out whether a new particle has collided with the cluster we check only its adjacent neighbors. This requires a special lattice which covers all particles and separates them into different cells. Doing so we check whether the particle collides or not only with particles from surrounding cells relative to a current particle position. We choose a cell size of this lattice to be equal to $32 R$. Second, using the lattice also simplifies and speeds up process of calculation of distance $d$ needed at 4-th step of algorithm. We mark all cells in the lattice as occupied or not and calculate $d$ based on only this information. This gives lower estimate on $d$. Details for this algorithm are presented in [7]. Third, we found that it is very effective for memory management to have particles being stored in cells.

\footnotetext{
${ }^{1}$ We refer to $R$ as the unit length equal to the radius of particles and to the length in the process of random walk realization.
}

One should be careful with system memory allocation procedures since memory allocation for new particles one by one will produce a great overhead and consume up to 5 times more memory. Each cell of the lattice has different number of particles in it and it is not reasonable to preallocate memory for all particles in cell because this number is unknown in advance. To deal with this we have implemented our own memory allocation routines which are very effective relative to computer memory requirements. Our own memory allocation procedures and special memory organization allows us to implement cell-based swap memory. As long as operating system swapping works with memory pages that are much larger than size of a cell with average number of particles in it, such large memory region will always contain particles that are on the boundary of the cluster and thus are always accessed by new coming walkers. Our cell-based swapping saves a single cell to a disk when the cell was not visited by new particles for a long enough time. Usage of swapping allows us to generate cluster with up to $200 \mathrm{mln}$ particles on a computer with only $2 \mathrm{~Gb}$ of RAM.

\section{Lattice algorithm and effect of local anisotropy}

Most of the improvements of the algorithm for generation of DLA clusters, described in the previous chapter, are consequences of its off-lattice nature and they are based on isotropy of random motion in plane. Still there exists a major class of objects that are either grown on lattice or have some intrinsic anisotropy. One can imagine a cluster build with atoms that form a lattice. Such a lattice is only being formed after the atoms have come into contact with each other. It is possible to model such features without essential changes in our algorithm if one adopts the so called antenna method [9, 10, 11]. Let particles constituting the cluster be only allowed to occupy a finite set of positions. This could be imagined as each particle of the cluster has $N_{f p}$ antennas in such a way that if new particle touches cluster at some point, it is shifted to the closest antenna carried by the parent particle. All antennas are aligned along the $N_{f p}$ directions (see Fig. 1). For example, the case of $N_{f p}=4$ with anisotropy directions perpendicular to each other is equivalent to square lattice model. And $N_{f p}=3$ represents hexagonal lattice. The only difference with real square lattice model is that the motion of a particle does not have any anisotropy in it until the particle is attached to the cluster.

In our study of DLA with anisotropy in particle attachment we add an extra parameter - adhesion of particles. With this property new particles stick to the cluster only after $N_{n r}$ collisions with the same antenna, instead of sticking after the first collision. Practically, this is the same as noise reduction in DLA, when each particle of cluster has a counter associated with it. When new particle hits the cluster, the counter is incremented. The particle is attached to the cluster only if the counter value is greater than some cut-off value $N_{n r}$, otherwise 


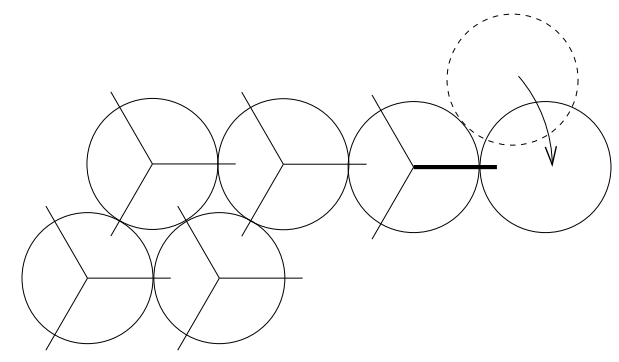

Figure 1: Antenna method for new particle attachment. Each new particle (dashed circle) moves to the closest antenna (bold line) of its parent particle.

the particle is destroyed and new one is released. One can think of the noise-reduction as of growth probability cut-off which eliminates lowest growth probabilities keeping only the most probable. It was a long time belief that noise reduction procedure results in faster convergence to asymptotic properties in DLA [12] since it allows only for most probable events to happen. In our simulation not each cluster particle but each antenna on each particle has its noise-reduction counter. This results in unexpected behavior of DLA properties which will be presented later in this paper.

\section{Measurement procedure}

The basic property of an ensemble of clusters that we are interested in is the cluster fractal dimension $D$. It is a common belief that fractal value of cluster dimension should be associated with some "criticality". Such approach is well developed for equilibrium processes and it is known that equilibrium criticality is generally described by set of two non-trivial exponents. In non-equilibrium and dynamical processes identification of criticality is still a challenging problem. Therefore, in the present paper we restrict ourselves with measurement of the value of well defined quantity - the cluster fractal dimension $D$.

Long-time correlations 6 and slow vanishing fluctuations [7] make it rather difficult to measure fractal dimension $D$ in standard way and with good precision. To overcome the difficulty, method of variable probe particle size was developed in Ref. [7]. The idea of this method is as follows. For each cluster we measure, for example, average deposition radius $R_{d e p, i}^{h m}(N, \delta)$ calculated as harmonicmeasure average with probe particles of size $\delta$ at cluster size $N$. With the help of scaling relation $N \propto R_{d e p, i}^{h m}{ }^{D_{i}}$ we extract the fractal dimension $D_{i}(N, \delta)$ of each cluster $i$ in ensemble as a function of $N$ and $\delta$. At the next step we average $D_{i}(N, \delta)$ over the ensemble: $D(N, \delta)=$ $1 / K \sum D_{i}(N, \delta)$ (see Fig. 2). And, finally, we take limit $\delta \rightarrow 0$ and find the quantity $D(N)=D(N, \delta=0)$ we are interested in. In order to find the limit $D(N, \delta \rightarrow 0)$ we have used in [7] the power law equation $D(N, \delta)=$ $D(N)+A \delta^{\beta}$. Although this equation fits well experimental data for $\delta>1$, its precision is not very good for

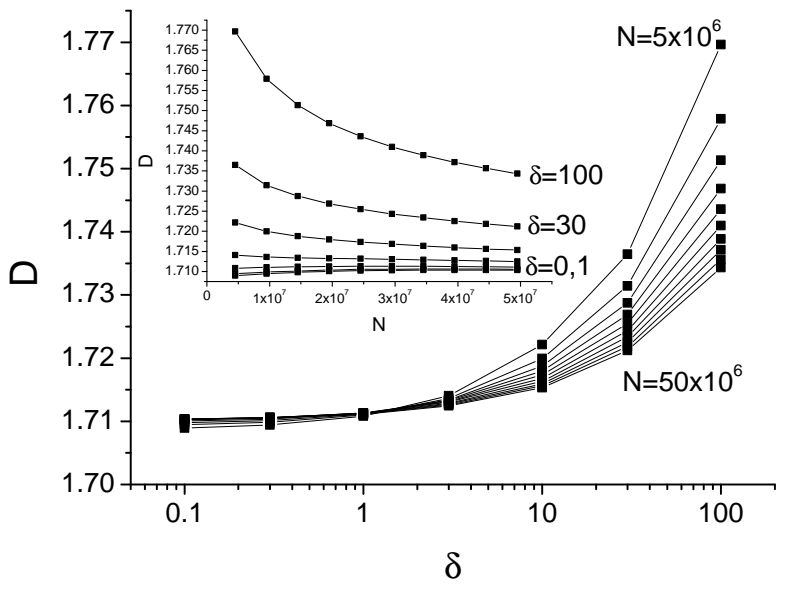

Figure 2: Dependence of fractal dimension $D(N, \delta)$ on size $N$ of the off-lattice cluster and on size $\delta$ of probe particles. Calculated over ensemble of 1000 clusters with $5 \cdot 10^{7}$ particles each.

$\delta \rightarrow 0$. Instead of the power law approximation used in [7], in this paper we use linear approximation for $D(N, \delta)$ for $\delta=0.1,0.3,1,3$. We have carried out the following check that confirms that the approximation is reasonable. For the calculation of $R_{d e p}^{h m}$ one needs to average position of probe particles that have touched the cluster at some point. One can choose to average either position of probe particle center or position of the point of contact. It is clear that in the limit $\delta \rightarrow 0$ resulting $D(N)$ should not depend on this choice. We have checked this in our simulations and found that our assumption holds quite well, i.e. linear approximation $D(N, \delta)=D(N)+A \delta$ could be used for calculation of $D(N)$.

Taking smaller probe particles gives them higher probability to penetrate deeper into cluster structure inside fjords. If the fjord structure is similar to rectangular slit of length $l$ and width $a$, then probability for a particle to pass through it is proportional to $\exp (-\pi l / a)$. Since particle of finite size $\delta$ affects effective channel width $a$, using infinitesimal size probe particles will allow greater part of cluster surface to be exposed thus resulting in higher accuracy of $D(N)$ estimation. Bottoms of fjords are screened strongly by branches and these regions belong to frozen part of the cluster. Taking them into calculation with slightly higher probability results in fractal dimension values with better statistical properties.

While we cannot treat the effect of variable probe particles analytically to explain that $D(N)$ obtained with the described procedure is a good quantity and extrapolates well the asymptotic fractal dimension of DLA, we propose the following empirical arguments. It is easy to see that curves $D(N, \delta)$ in Fig. 2 are very similar, and it is actually possible to merge them into a single curve. For an infinitely large cluster, i.e. for $N=\infty$, the finite size of particles would be unimportant. But for finite cluster size 


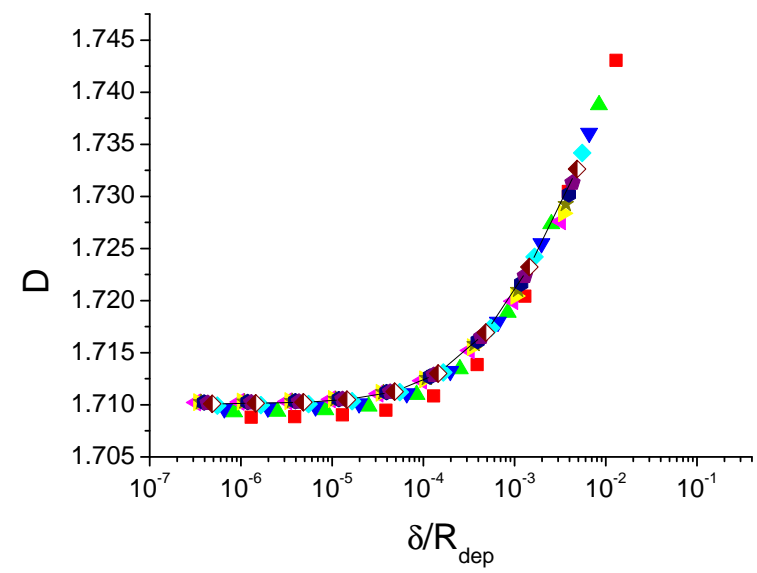

Figure 3: (Color online) Rescaled $D(N, \delta)$ dependence (shown in Fig. 2 ) in the form $D\left(\delta / R_{\text {dep }}(N)\right)$. Different colors represent different cluster sizes $N$.

we should take size of particles into account. In addition to the size of the cluster there exists only one more natural length scale - the particle size. Although we cannot change size of particles in cluster because all lengths are proportional to this quantity, we can change size of particles during the measurement procedure. This reasoning leads us to the idea to rescale $\delta$ parameter in $D(N, \delta)$ dependence in the following form: $D\left(\delta / R_{d e p}^{h m}(N)\right)$. Surprisingly, after this transformation all the curves corresponding to different cluster sizes merge into a single curve, as seen in Fig. 3. It is also natural to rescale the size of probe particle in this form since the probability for a particle to pass through the fjord is proportional to the difference in fjord width and particle size, and the width of the fjord is directly proportional to the $R_{d e p}$, if we assume that the number of branches does not change with the time. In other words, there are only two independent length scales $\delta$ and $R_{\text {dep }}$. The existence of such scaling means that if we take probe particles ten times smaller than usual the fractal dimension will be the same as for the cluster that has $R_{d e p}$ ten times bigger and is measured with probe particles of unit size. Thereby, measuring fractal dimension of DLA in the limit of probe particles of vanishing size results in values converging faster to the asymptotic thermodynamic regime.

\section{Results}

We have generated clusters with different number $N_{f p}$ of axes of the anisotropy field, varying from $N_{f p}=3$ to 8 , as well as off-lattice clusters (this case is equivalent to $\left.N_{f p} \rightarrow \infty\right)$. Each ensemble has 1000 clusters and each cluster consists of 50 millions of particles.

Fractal dimension of the clusters, calculated with the help of technique described in Sec. 4, is presented in Figs. 4 and 5. Fractal dimension $D(N)$ for clusters with 3 axes

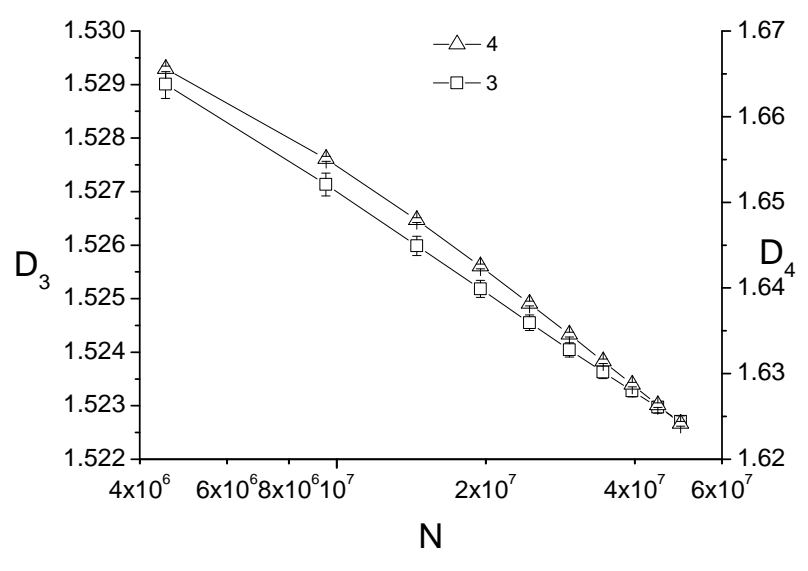

Figure 4: Fractal dimension $D(N)$ of clusters grown with $N_{f p}=3$ $\left(D_{3}\right.$, left axis) and $4\left(D_{4}\right.$, right axis) anisotropy axes as a function of cluster size $N$.

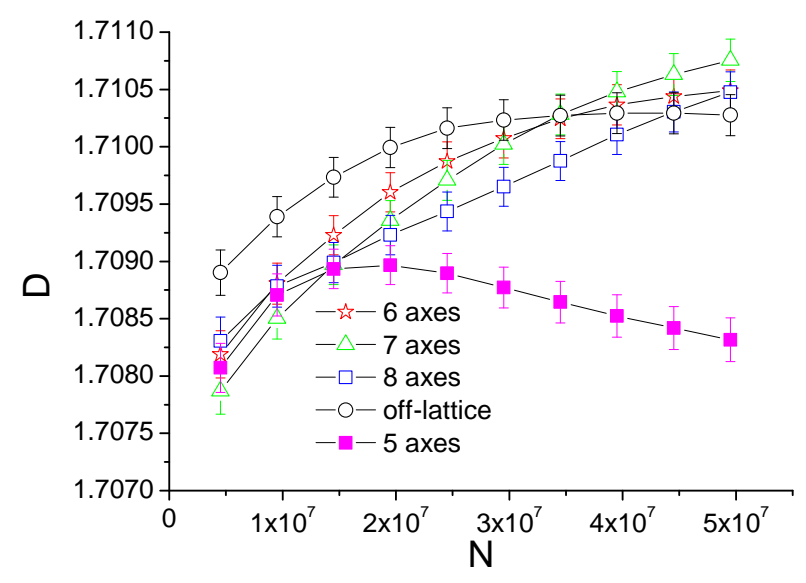

Figure 5: (Color online) Fractal dimension $D(N)$ of clusters grown with $N_{f p}=5,6,7,8$ anisotropy axes and also of off-lattice clusters as a function of cluster size $N$.

(hexagonal lattice) diminishes with increasing the size of the cluster as $D_{3}(N)=1.5699(14)-0.00613(19) \log _{10} N$; for clusters with 4 axes (square lattice) $D_{4}(N)=1.9400(22)-$ $0.04093(30) \log _{10} N$. Therefore, for big enough clusters fractal dimension of on-lattice models goes down. According to prediction of Kesten, the lowest possible fractal dimension for such objects is exactly $3 / 2$ [13]. However, to reach this value one needs to generate cluster with at least $10^{11}$ particles. This value is 3 orders of magnitude higher than value reachable with modern computers and algorithms.

In contrast to the previous case, fractals with $N_{f p}>5$ tend to have fractal dimension $D$ equal to dimension of off-lattice clusters. The closer the branches of DLA are located, the higher are screening effects and the higher are fluctuations that destroy anisotropy. 


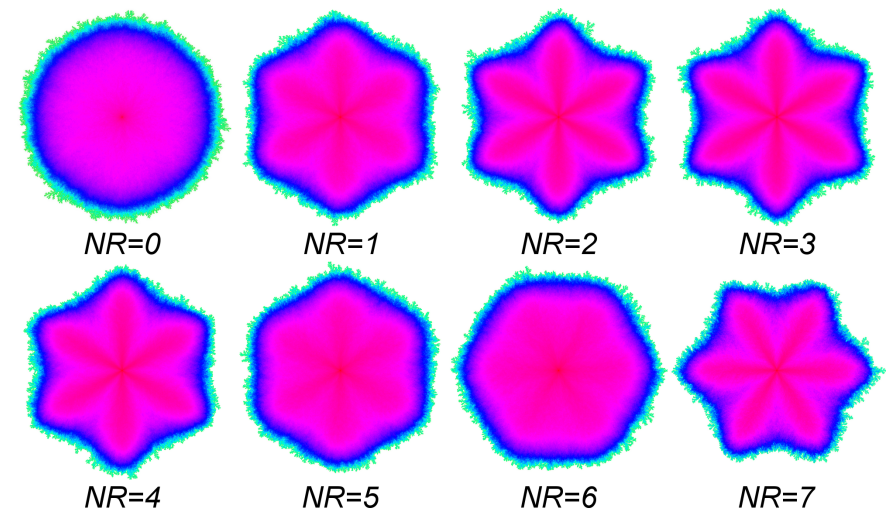

Figure 6: (Color online) Average density of particles in ensemble of clusters grown with six anisotropy axes with varying noise reduction level $N_{n r}=0,1,2,3,4,5,6,7$.

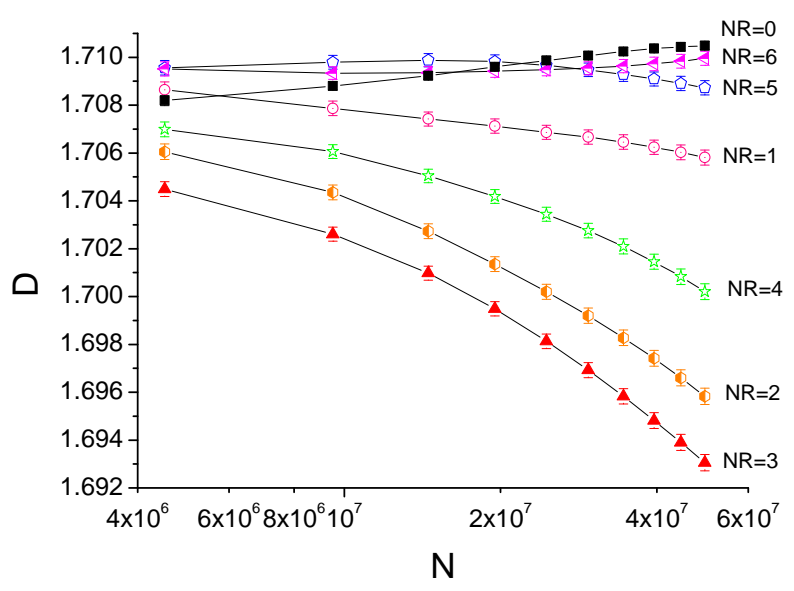

Figure 7: (Color online) Fractal dimension $D(N)$ of clusters grown with $N_{f p}=3$ anisotropy axes and varying noise reduction level as a function of cluster size $N$.

Change in $N_{f p}$ anisotropy parameter not only influences fractal dimension of the cluster but also results in transition in the geometrical structure of DLA. Clusters with $N_{f p}=3,4$ (hexagonal and square lattices) prefer to grow along anisotropy axes of the cluster. The number of main branches for such clusters is equal to $N_{f p}$. Clusters with $N_{f p}>5$ look isotropic on the average and do not have any preferred growth directions. Peculiar case $N_{f p}=5$ separates these two well-defined regions. Asymptotic behavior in that case is obscured by slow transition effects. Looking at Fig. 5 one may suggest that in the limit $N \rightarrow \infty$ the asymptotic value of the fractal dimension $D_{5}$ will be $3 / 2$. It is worthwhile to mention that on the average clusters with $N_{f p}=5$ also prefer to grow along several preferred directions, as in $N_{f p}=3,4$ case. However, in contrast to the case $N_{f p}=3,4$, these directions are not alongside with anisotropy axes but are along the axes turned by the angle $\pi /\left(2 N_{f p}\right)$, i.e. they are strictly between the anisotropy axes.
We also analyze influence of the noise reduction on the structure of the cluster. Let us start first with $N_{f p}=6$ case. Average density of particles for clusters with six anisotropy axes is presented in Fig. 6, where average was taken over ensemble of 1000 clusters. The parameter $N_{n r}$ represents the noise reduction level used to grow an ensemble of clusters. The case $N_{n r}=0$ corresponds to the usual simulation without noise reduction, $N_{n r}=1$ means that particle will be attached on the second collision with the particular antenna, etc. Clusters without noise reduction are isotropic and symmetric. Anisotropy decrease becomes more pronounced with increasing the noise reduction, however preferred growth directions of cluster are no more aligned with the antenna directions ${ }^{2}$ Further increase of $N_{n r}$ makes cluster more asymmetric and at $N_{n r}=6$ main branches of the cluster are being rotated by the angle $\pi /\left(2 N_{f p}\right)$.

Alteration in geometrical structure is also reflected on fractal dimension of the system. This could be seen from the Fig. 6. As soon as the cluster becomes anisotropic, its fractal dimension decreases until $N_{n r}=3$. After that its structure tends to be more symmetric $\left(N_{n r}=5\right)$ until rapid change in preferred growth directions at $N_{n r}=6$ occurs. Further increase in value of $N_{n r}$ leads to decrease in $D(N)$ with size of the cluster while aggregate structure always stays asymmetric and its branches grow along anisotropy directions.

\section{Morphological diagram and phase transition}

Based on the properties of clusters presented in the previous section we propose the following picture that separates all two-dimensional DLA-like objects into two major classes. Clusters with low number of anisotropy growth directions $N_{f p}<6$ and clusters with large number of growth direction but grown with noise-reduction procedure $N_{n r}>N_{n r}^{c r i t}\left(N_{f p}\right)$ have anisotropic shape with number of main branches equal to $N_{f p}$. Their fractal dimension is $3 / 2$ in the thermodynamic limit of big enough systems. Another class of objects consists of structures that look symmetrical on the average with no well-defined preferred direction. Their fractal dimension approaches to the well known value of 1.710 corresponding to off-lattice DLA clusters. We sketch this result in the diagram shown in Fig. 8 ,

\section{Results and discussion}

Based on the intensive numerical simulations we propose morphological diagram for two-dimensional structures which may be typical for all processes where diffusion dominates growth. At first glance similar behavior was found

\footnotetext{
${ }^{2}$ The first antenna (anisotropy axis) is always aligned along $\mathrm{X}$ axis in all figures.
} 


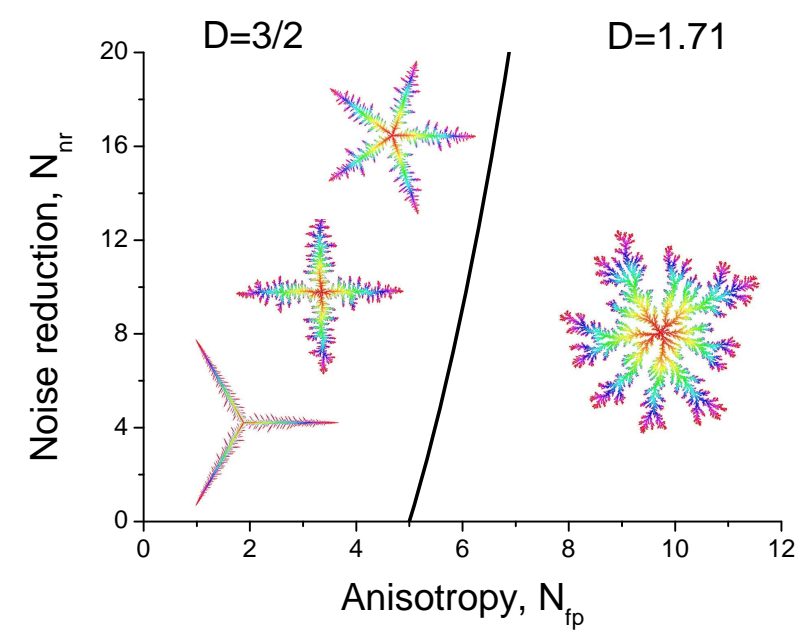

Figure 8: (Color online) Morphological diagram of DLA clusters. The region left from the solid line corresponds to the $N_{f p}$-fold fractal crystals with asymptotical fractal dimension $3 / 2$. The region to the right of the solid line corresponds to the random fractal with asymptotic fractal dimension 1.710. Point $\left(N_{f p}=5, N n r=0\right)$ is marginal point.

in paper by Nittmann and Stanley [12 for six-fold symmetry structures grown with DBM algorithm. But in contrast to [12] our simulation for clusters with $N_{f p}=3,4,5$ results in fractal dimension equal to 1.5 , while Nittmann and Stanley propose that it is equal to 1.71. Also finite noise reduction in our simulation could change structure of the aggregate from one class to another in contrast to [12]. In our model anisotropy is not required to be specially enhanced to be noticeable.

In the paper by Aukrust and others [14 authors have also proposed morphological transition taking place when the level of anisotropy was varied. But authors have studied only square-lattice model where anisotropy was varied with number of local neighbors.

In our simulation we have focused on effects caused by underlying lattice anisotropy for different lattices $\left(N_{f p}=\right.$ 3,4 ) and pseudo-lattices $N_{f p}>5$. Since $N_{f p}=5$ is the marginal case that separates $N_{f p}$-fold clusters and fractal clusters, we also propose that the number of main branches in DLA is somewhere between 5 and 6 . This number is slightly higher than 4.8 predicted by Ball [1] but is still very close to that value.

A. Yu. Menshutin thanks Dynasty Foundation for supporting this work.

\section{References}

[1] N. Goldenfeld, Lectures on Phase Transitions and the Renormalization Group (Addison-Wesley, Reading, 1992).

[2] T.A. Witten and L.M. Sander, Phys. Rev. Lett. 47 (1981) 1400.

[3] A.A. Shibkov, et al, J. Cryst. Growth 285 (2005) 215; B. Chopard, H. J. Herrmann, and T. Vicsek, Nature 353 (1991) 409; M. Matsushita, et al, Biofilms 1 (2004) 305; S. Ogura, et al, Phys. Rev. B 73 (2006) 125442; Z. Saghi, X. Xu, and G. Möbus, Phys. Rev. B 78 (2008) 205428; Frank-J. Meyer zu Heringdorf, M. C. Reuter and R. M. Tromp, Nature 412 (2001) 517; O. Praud and H. L. Swinney, Phys. Rev. E 72 (2005) 011406; C. Andersson, S. Rasmussen, and R. White, Environment and Planning B: Planning and Design 29 (2002) 841.

[4] B. Mandelbrot, Physica A 191, 95 (1992).

[5] A. Bunde and S. Havlin, eds., Fractals and Disordered Systems (Springer, Berlin, 1996).

[6] A. Yu. Menshutin, L. N. Shchur, Phys. Rev. E 73, 011407 (2006).

[7] A.Yu Menshutin, L.N. Shchur, V.M. Vinokur, Phys. Rev. E 75 (2007) 010401 (R); Physica A 387 (2008) 6299.

[8] R.M. Ziff, S.N. Majumdar and A. Comtet, J. Chem. Phys. 130 (2009) 204104.

[9] P. Meakin, Phys. Rev. A 33 (1986) 3371

[10] J.P. Eckmann, P. Meakin, I. Procaccia and R. Zeitak, Phys. Rev. Lett. 65 (1990) 52.

[11] R.C. Ball, Physica A 140 (1986) 62.

[12] J. Nittmann and H.E. Stanley, Nature 321 (1986) 633.

[13] H. Kesten, Stoch. Proc. and Appl. 25 (1987) 165.

[14] T. Aukrust, M.A. Novotny, D.A. Browne and K. Kaski, Phys. Rev. A 39 (1989) 2587. 